\title{
Utilização do método de Newton-Raphson para análise de planos de financiamento no centro comercial de Abaetetuba-PA
}

\section{Use of the Newton-Raphson method to analyze financing plans in the Abaetetuba-PA shopping center}

\author{
Manuel de Jesus dos Santos Costa ${ }^{1 *}$, Rômulo Correa Lima ${ }^{1}$, José Francisco da Silva Costa ${ }^{1}$
}

\begin{abstract}
RESUMO
O objetivo deste trabalho foi trazer uma análise da viabilidade de planos de financiamento para bens de consumo no centro comercial do município de Abaetetuba, localizado no estado do Pará, com auxílio da matemática numérica atrelada a um ambiente computacional. Para tanto foi realizada uma pesquisa no centro comercial do referido município, no intuito de coletar dados de produtos que possuíssem planos de financiamento distintos, nos quais o consumidor tem possibilidades de fazer escolhas de um financiamento que lhe seja mais vantajoso. Neste contexto, apresentou-se um modelo matemático representando a situação problema real vinculada a planos de pagamentos diferenciados de um computador, um micro-ondas e uma televisão. Em seguida, utilizou-se o método de Newton - Raphson para resolver a equação oriunda do modelo supracitado, onde procedimentos analíticos não se aplicam. Ao analisar-se os resultados, foi possível concluir qual o plano indicava ser mais vantajoso financeiramente para o consumidor.
\end{abstract}

Palavras-chave: Newton-Raphson; Financiamento; Taxa de juros.

\section{ABSTRACT}

The objective of this work was to analyze the feasibility of financing plans for consumer goods in the commercial center of the city of Abaetetuba, located in the state of Pará, with the help of numerical mathematics linked to a computational environment. For this purpose, a survey was carried out in the commercial center of that municipality, in order to collect data on products that had different financing plans, in which the consumer is able to make choices about financing that is more advantageous to him. In this context, a mathematical model representing the real problem situation linked to different payment plans of a computer, a microwave and a television was presented. Then, the Newton - Raphson method was used to solve the equation derived from the aforementioned model, where analytical procedures do not apply. By analyzing the results, it was possible to conclude which plan indicated to be the most financially advantageous for the consumer.

Keywords: Newton-Raphson; Financing; Interest rate.

\footnotetext{
${ }^{1}$ Universidade Federal do Pará-UFPA.

*E-mail: manuelsc@ufpa.br
} 


\section{INTRODUÇÃO}

Uma situação rotineira que se identifica na vida dos consumidores brasileiros são as prestações adquiridas no ato de uma compra, pois nem sempre o comprador possui o montante necessário para realizar a aquisição de um determinado bem de consumo. Este cenário indica a vulnerabilidade do consumidor, no que diz respeito a um apoio técnicocientífico para obter a informação de qual é a cobrança de juros, principalmente se não houver no procedimento de compra uma declaração explicita de tal cobrança, vinculado ao fato da presença de agentes e não consultores de venda, os quais objetivam apenas vender o produto.

Assim, se o usuário possuir opções de compra em determinadas lojas sem o devido esclarecimento, o mesmo encontrar-se-á inviabilizado de escolher o plano mais vantajoso, ficando restrito ao processo de retirar comparação direta do valor final específico de cada plano, podendo escolher desta maneira o plano que apresentar o menor valor final, o que não garante ao mesmo a melhor taxa de juros. No entanto, o que rege esta conclusão de forma consistente e técnica não é o montante final de cada plano, mas sim o conhecimento da menor taxa de juros vinculada ao mesmo, a qual deverá ser considerada pelo consumidor.

Diante do exposto, pretende-se através deste trabalho, disponibilizar uma estratégia que possibilite ao consumidor o direito de escolha, não mais restrito apenas à possibilidade de poder executar o pagamento total da compra efetuada, mas também que lhe seja vantajoso financeiramente no que tange à taxa de juros incluída no valor do financiamento.

Assim, com a finalidade de contribuir para uma escolha consistente do consumidor, em particular do município de Abaetetuba-PA, a presente pesquisa será baseada em um modelo matemático vinculado a uma solução numérica estimada pelo método de Newton-Raphson, o qual se propõe estimar a raiz de uma equação a menos de uma precisão que o problema requeira. A escolha deste método deve-se a uma ampla aplicabilidade como ferramenta auxiliar na solução de diversos problemas de Ciências e de Engenharia.

Por conseguinte, objetivando disponibilizar ao consumidor uma melhor forma de pagar um determinado produto, quando este encontra-se diante de uma situação na qual são oferecidos dois ou mais planos de pagamento, sobre os quais em geral não se possui informações de suas taxas de juro. Assim, será desenvolvida uma estratégia baseada em 
uma pesquisa no centro comercial de Abaetetuba-PA composta por eletrodomésticos e eletrônicos que ofereciam no momento planos com formas de pagamentos diferentes e não explicitavam detalhadamente tais planos.

De posse desses dados será analisado como pode-se chegar a uma situação de indicação qual plano é mais vantajoso financeiramente. Para tanto, executando o procedimento desenvolvido na pesquisa para um produto que possui duas formas de pagamento, será estimado os juros para cada plano, indicando qual deles é mais vantajoso financeiramente para o consumidor.

\section{MODELO MATEMÁTICO PARA APLICAÇÃO}

Um modelo matemático para estimar a taxa de juros de uma determinada compra a prazo será utilizado, adotando a metodologia que o Programa de Proteção e Defesa do Consumidor (PROCON) empregada para calcular as taxas de juros cobradas nas compras parceladas como abordado por Ceccatto (2009). Neste sentido, seja:

$$
\begin{aligned}
& V P-\text { Valor a financiar } \\
& P_{j} \text { - Parcelas, onde } j=1,2,3, \cdots, n . \\
& i \text { - Taxa de juros } \\
& n \text { - Prazo ou } \mathrm{n}^{\mathrm{o}} \text { de parcelas }
\end{aligned}
$$

Agora, considerando:

$$
V P=P_{1}+P_{2}+P_{3}+\cdots+P_{n} .
$$

Como ocorre a cobrança de juros compostos vinculados a uma determinada taxa $(i)$ em cada $P_{j}$ tem-se (PUCCINI, 1993):

$$
\begin{gathered}
P_{1}(1+i) \\
P_{2}(1+i)^{2} \\
P_{j}(1+i)^{n} .
\end{gathered}
$$

Neste contexto, considerando-se parcelas fixas $(P)$, logo:

$$
P_{1}(1+i)=P
$$

implica: 


$$
P_{1}=\frac{P}{1+i}
$$

ou ainda,

$$
V P=P\left(\frac{1}{1+i}+\frac{1}{(1+i)^{2}}+\frac{1}{(1+i)^{3}}+\cdots+\frac{1}{(1+i)^{n}}\right)
$$

Assim, observa-se que no interior dos parênteses de (8), define a soma de uma progressão geométrica (PG), onde seu primeiro termo $a_{1}$ e sua razão $q$ é determinado por:

$$
a_{1}=q=\frac{1}{1+i}
$$

Portanto, considerando a soma finita de uma P.G (BARRETO FILHO e SILVA, 2003):

$$
S=\frac{a_{1}\left(q^{n}-1\right)}{q-1}
$$

e, inserindo (9) em (10), tem-se:

$$
\begin{gathered}
S=\frac{\left(\frac{1}{1+i}\right)\left[\left(\frac{1}{1+i}\right)^{n}-1\right]}{\frac{1}{1+i}-1}, \\
S=\frac{\left(\frac{1}{1+i}\right)^{n+1}-\frac{1}{1+i}}{\frac{1-(1+i)}{1+i}} \\
S=\frac{\left(\frac{1}{1+i}\right)^{n+1}-\frac{1}{1+i}}{-\frac{i}{1+i}}
\end{gathered}
$$

Dessa forma, multiplicando (13) por $(1+i)$, tem-se:

$$
S=\frac{1-(1+i)^{-n}}{i}
$$

Logo, substituindo (14) em (8), obtém-se:

$$
\frac{V P}{P}=\frac{1-(1+i)^{-n}}{i}
$$


Para viabilizar a implementação do método de Newton-Raphson utilizando a formulação (15), faz-se uma mudança de variável, onde haverá uma incógnita que caracterizará o zero (raiz) de uma determinada função, fato este relevante para alcançar o propósito da pesquisa.

Assim, considerando,

$$
x=1+i
$$

e fazendo $\frac{V P}{P}=m_{1}\left(^{*}\right)$ em (15), tem-se:

$$
m_{1}=\frac{1-x^{-n}}{x-1}
$$

Em seguida, multiplicando em ambos os membros de (17) por $x^{n}$, obtém-se:

$$
\begin{gathered}
m_{1} x^{n}=\frac{1-x^{-n}}{x-1} x^{n}, \\
m_{1} x^{n}=\frac{x^{n}-1}{x-1}, \\
m_{1} x^{n}(x-1)=x^{n}-1 .
\end{gathered}
$$

Portanto, fazendo (20) igual a zero, ou seja, $f(x)=0$, determina-se:

$$
\begin{gathered}
f(x)=m_{1} x^{n}(x-1)-x^{n}+1, \\
f(x)=m_{1} x^{n+1}-m_{1} x^{n}-x^{n}+1, \\
f(x)=m_{1} x^{n+1}-\left(m_{1}+1\right) x^{n}+1=0,
\end{gathered}
$$

onde esta última define uma equação algébrica de grau $n+1$. Verifica-se que:

$$
x=1
$$

caracteriza uma raiz para (23), porém esta não apresenta significado para o problema em análise, uma vez que, pela formulação (16), quantifica a taxa de juros nula. Dessa forma, faz-se necessário, encontrar um outro valor para $x$, onde $f(w)=0$. Neste sentido, uma vez encontrado tal valor será possível calcular o juro até então desconhecido. Para isso será necessário utilizar uma estratégia numérica uma vez que o modelo matemático apresentado gera uma equação que pode apresentar um grau elevado, pois o mesmo está diretamente dependente do número de parcelas do financiamento. Dessa maneira, a 
metodologia que será utilizada é composta pelas fases de isolamento da raiz e a estimativa da mesma a menos de uma precisão requerida

\section{ISOLAMENTO DA RAIZ (FASE I)}

O processo utilizado para isolar a raiz de uma determinada equação $f(x)=0$, seguirá a seguinte estratégia: a partir de $f(x)=0$, obtém-se uma identidade $g(x)=$ $h(x)$, em seguida faz-se o esboço dos gráficos das funções $g(x)$ e $h(x)$ no mesmo sistema de eixos, localizando o ponto $x=\xi$ onde as curvas se interceptam, determinado $f(\xi)=$ $0 \Leftrightarrow g(\xi)=h(\xi)$, isolando a raiz em questão (Gráfico 1).

Gráfico 1 - Representação geométrica da localização de uma determinada raiz $\xi$

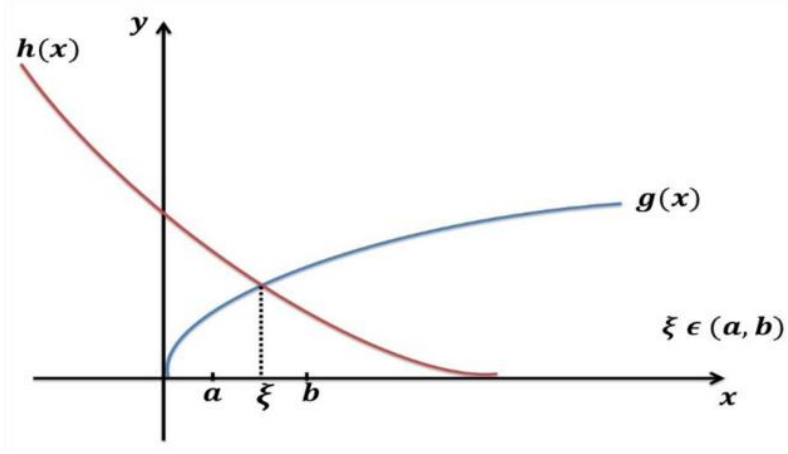

Fonte: Adaptado de RUGGIERO, 1996, p.36

\section{ESTIMATIVA DA RAIZ (FASE II)}

Consiste na utilização de um método iterativo que segue uma sequência de instruções que são executadas passo a passo. Estas instruções por sua vez têm etapas que são repetidas sistematicamente tornando-a um ciclo, até que se atinja uma precisão préestabelecida. Esta repetição é chamada de iteração. Portanto, cada iteração executada obtém-se um resultado; se este resultado estiver satisfazendo à aproximação requerida da raiz exata, a iteração para, sendo finalizado o cálculo, caso contrário o processo reinicializa-se a partir do resultado encontrado anteriormente até que se obtenha uma aproximação mais acurada da raiz exata da equação em questão.

Tal procedimento está representado através de um diagrama de fluxo (ver, Figura 1) (RUGGIERO, 1996, p. 38). Existem uma gama de métodos iterativos que se propõem a estimar o valor numérico de uma raiz vinculada a uma equação do tipo $f(x)=0$, entretanto neste trabalho será utilizado o método de Newton-Raphson. 
Figura 1- Diagrama de fluxo de um processo iterativo

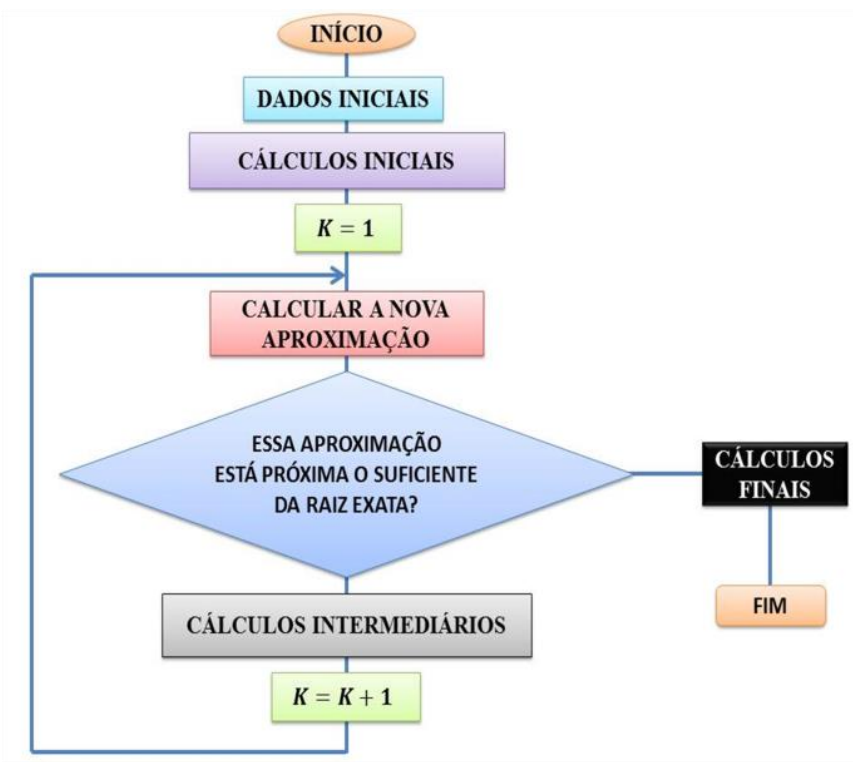

Fonte: Adaptado RUGGIERO, 1996, p. 38

\section{MÉTODO DE NEWTON-RAPHSON}

Seja $f(x)$ uma função continua no intervalo $[a, b]$ e $\xi$ a sua única raiz neste intervalo; as derivadas $f^{\prime}(x)\left(f^{\prime}(x) \neq 0\right)$ e $f^{\prime \prime}(x)$ devem também ser contínuas. Neste contexto, a fim de obter-se uma aproximação $x_{0}$ da raiz $\xi$ cada vez mais acurada, traçase a partir do ponto $B_{0}\left[x_{0}, f\left(x_{0}\right)\right]$, uma reta tangente à curva $y=f(x)$, que intercepta 0 eixo dos $x$ no ponto $x_{1}$. Do ponto $B_{1}\left[x_{1}, f\left(x_{1}\right)\right]$ traça-se outra reta tangente à curva que corta o eixo dos $x$ no ponto $x_{2}$, sendo este ponto uma melhor aproximação da raiz. $\mathrm{O}$ processo se repete até que se encontre $\xi=x_{n}$ com uma exatidão pré-fixada (Gráfico 2). 
Gráfico 2-Representação gráfica do método de Newton-Raphson

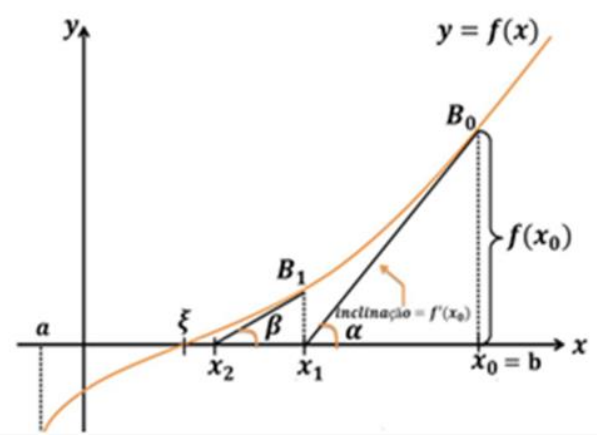

Fonte: Adaptado de RUGGIERO, 1996, p.36

$$
\begin{gathered}
\operatorname{tg}(\alpha)=\frac{f\left(x_{0}\right)}{x_{0}-x_{1}}=f^{\prime}\left(x_{0}\right) \\
x_{0}-x_{1}=\frac{f\left(x_{0}\right)}{f^{\prime}\left(x_{0}\right)} \\
x_{1}=x_{0}-\frac{f\left(x_{0}\right)}{f^{\prime}\left(x_{0}\right)} \\
\operatorname{tg}(\beta)=\frac{f\left(x_{1}\right)}{x_{1}-x_{2}}=f^{\prime}\left(x_{1}\right) \\
x_{1}-x_{2}=\frac{f\left(x_{1}\right)}{f^{\prime}\left(x_{1}\right)} \\
x_{2}=x_{1}-\frac{f\left(x_{1}\right)}{f^{\prime}\left(x_{1}\right)} .
\end{gathered}
$$

Por indução, obtém-se a fórmula analítica do método Newton - Raphson, como segue (BARROSO, 1987):

$$
x_{n+1}=x_{n}-\frac{f\left(x_{n}\right)}{f^{\prime}\left(x_{n}\right)} \quad n=0,1,2,3, \ldots
$$

\section{Escolha de $x_{0}$}

O $x_{0}$ é um dos extremos do intervalo $[a, b]$ que contém a raiz, sendo assim, para que seja deduzido o valor inicial $x_{0}$, verifica-se qual valor dos extremos satisfaz a seguinte desigualdade (BARROSO, 1987):

$$
f\left(x_{0}\right) \cdot f^{\prime \prime}\left(x_{0}\right)>0
$$

Critério de parada

Um método iterativo consiste em repetir sistematicamente o processo de iteração até atingir a precisão requerida. Assim, para obter-se uma raiz com uma determinada exatidão será utilizado o seguinte critério (BARROSO, 1987): 


$$
\frac{\left|x_{n}-x_{n-1}\right|}{\left|x_{n}\right|} \leq \varepsilon \text { (tolerância) }
$$

onde $n=1,2,3, \ldots$, com $\varepsilon$ sendo uma precisão pré-estabelecida, sendo $x_{n-1}$ e $x_{n}$ raízes encontradas sequencialmente nesta ondem. Portanto se as raízes $x_{n-1}$ e $x_{n}$ satisfazerem o critério da precisão estabelecido considera-se $x_{n}=\xi$.

\section{LÓCUS DA PESQUISA}

O município de Abaetetuba, localizado no nordeste do Estado do Pará apresenta em seu setor comercial uma ampla rede de estabelecimentos das mais diversas atividades, quais sejam, lojas de calçados, eletrodomésticos, eletroeletrônicos, acessórios em geral (Figura 2), estando assim o consumidor sempre sujeito a várias opções relacionadas a compra de um determinado produto. No entanto, sem uma análise técnica a priori, este consumidor estará sujeito a uma escolha equivocada no que concerne a um plano aparentemente mais vantajoso financeiramente.

Figura 2- Município de Abaetetuba e atividade comercial

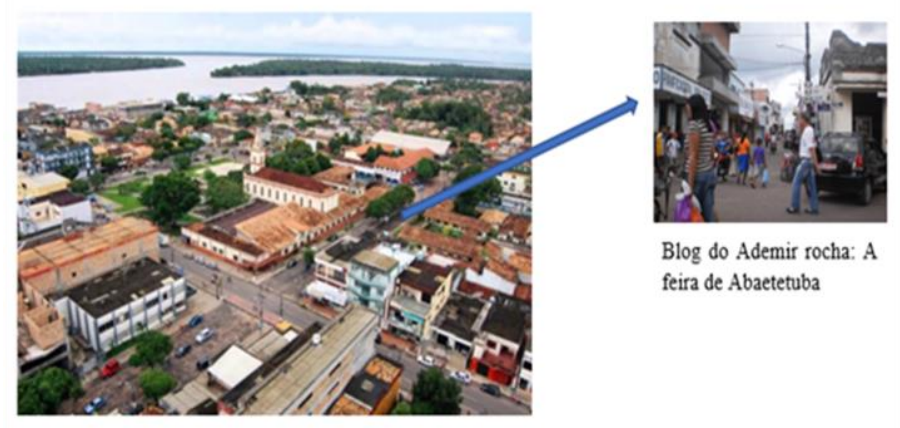

Fonte: Adaptado de RUGGIERO, 1996, p.36

\section{RESULTADOS E DISCUSSÕES}

Nesta seção serão apresentados os planos de financiamento de produtos, obtidos através de uma pesquisa realizada no centro comercial de Abaetetuba-PA, tendo como universo de amostragem os setores de eletrônicos e eletrodomésticos, a fim de inserir tais dados reais no modelo matemático apresentado, foram coletados dados nas lojas A, B e C referentes a 3 (três) produtos, respectivamente, sendo que os planos dos produtos estão descritos nos quadros (1), (2) e (3). Segue abaixo os quadros dos planos de pagamentos 
registrados, onde os dados são inseridos em (*) e (23), obtendo as equações vinculadas a cada plano de financiamento.

Quadro 1 - Planos de financiamento de um computador na loja A

\begin{tabular}{|c|c|}
\hline Á vista de & $\mathrm{R} \$ 1.107,00$ \\
\hline Plano A & Entrada de $402,00+5$ de $\mathrm{R} \$ 167,00$ \\
\hline Plano B & Entrada de $600,00+5$ de $\mathrm{R} \$ 126,00$ \\
\hline
\end{tabular}

Fonte: Pesquisa de campo

\section{Plano A}

$P=5$

$m_{1}=\frac{V P}{P} \Rightarrow \frac{1107-402}{167}=4,222$

$f(x)=4,222 x^{6}-5,222 x^{5}+1=0$

\section{Plano B}

$P=5$

$m_{1}=\frac{V P}{P} \Rightarrow \frac{1107-600}{126}=4,024$

$f(x)=4,024 x^{6}-5,024 x^{5}+1=0$

De forma análoga, os dados registrados para os demais produtos foram utilizados nas formulações matemáticas mencionadas anteriormente, obtenso-se:

Quadro 2 - Planos de financiamento de um micro-ondas na loja B

\begin{tabular}{|c|c|}
\hline Á vista de & $\mathrm{R} \$ 400,50$ \\
\hline Plano A & Entrada de 200,00 + 9 de R \$ 27,10 \\
\hline Plano B & Entrada de 300,00 + 4 de R \$ 36,25 \\
\hline
\end{tabular}

Fonte: Pesquisa de campo

\section{Plano A}

$f(x)=7,399 x^{10}-8,399 x^{9}+1=0$
Plano B

$f(x)=2,772 x^{5}-3,772 x^{4}+1=0$ 
Quadro 3 - Planos de financiamento de uma televisão na loja C

\begin{tabular}{|c|c|}
\hline Á vista de & $\mathrm{R} \$ 981,00$ \\
\hline Plano A & Entrada de 400,00 + 9 de R \$ 77,66 \\
\hline Plano B & Entrada de 500,00 + 9 de R \$ 64,55 \\
\hline
\end{tabular}

Fonte: Pesquisa de campo

Plano A

$(x)=7,481 x^{10}-8,481 x^{9}+1=0$
Plano B

$f(x)=7,452 x^{10}-8,452 x^{9}+1=0$

\section{ANÁLISES NUMÉRICAS DOS PLANOS COMERCIALIZADOS}

Tendo em vista os resultados anteriores, questiona-se. Qual plano de financiamento relacionado a um dado produto mostra-se mais vantajoso financeiramente para o consumidor? Para responder este questionamento, foi utilizado a modelagem apresentada anteriormente em conjunto com o método de Newton-Raphson. Para efetivar os cômputos, bem como a produção dos gráficos apresentados, utilizou-se um auxilio computacional. Tal estratégia proporcionou identificar qual financiamento apresenta a menor taxa de juros e consequentemente definir qual financiamento é mais vantajoso para o consumidor. A seguir, será apresentado os resultados obtidos para cada produto vinculado a um determinado financiamento.

I- Produto - Computador: dados vinculados aos planos registados no Quadro (Quadro 1).

Plano A - Entrada de 402,00 + 5 de R $\$ 167,00$

Apresenta-se novamente a equação que modela o plano A, ou seja,

$$
f(x)=4,222 x^{6}-5,222 x^{5}+1=0
$$

Para determinar o resultado almejado, a modalidade de financiamento necessita passar pelas fases I e II. Na fase I o processo utilizado para isolar a raiz da equação pode ser visualizado geometricamente no Gráfico (3), assim, fazendo $f(x)=0$,obtém-se,

$$
h(x)=4,222 x^{6} \text { e } k(x)=5,222 x^{5}-1 .
$$


Produzindo os gráficos das funções $h(x)$ (cor, ciano) e $k(x)$ (cor, vermelha) no mesmo sistema de eixos, pode-se localizar a raiz dentro do intervalo [1.01,1.2]. Neste contexto, aplica-se a condição (32), definindo desta maneira a aproximação inicial da raiz $x_{0}=1.2$. Em seguida, aplica-se a fase II que consiste na utilização do método de NewtonRaphson definido por (31) para estimar a raiz procurada, que neste caso considera-se uma precisão $\varepsilon=10^{-7}$. Desta forma, o valor aproximado da raiz determinada foi de $\xi_{A}=1.059199150190583$, a qual inserida em (16), obtém-se a taxa de juros $i_{A}=0,0591$ ou $i_{A}=5,91 \%$ ao mês (Gráfico 4$)$.

Gráfico 3 - Isolamento da raiz necessário para estimar a taxa de juros vinculada ao Plano A de financiamento (computador)

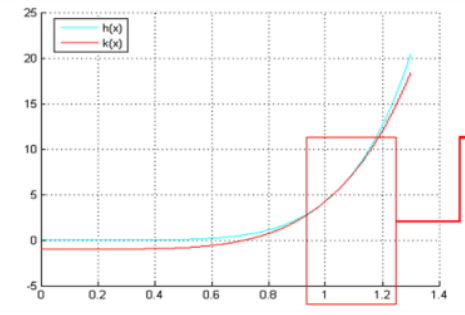

Fonte: Autoria própria

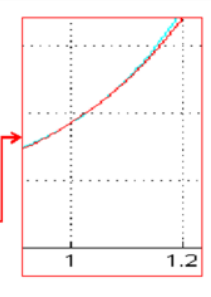
Gráfico 4 -Valor da raiz vinculada a modelagem
do plano A de financiamento (computador)

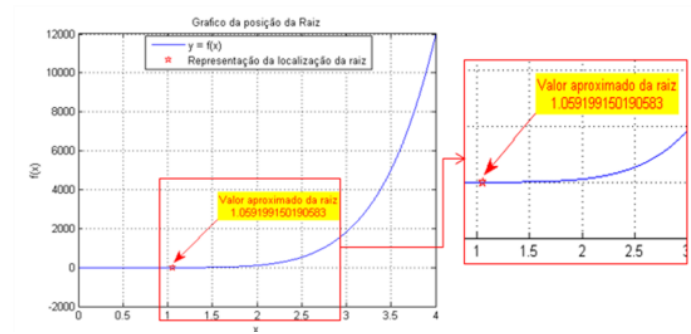

Fonte: Autoria própria

Procedendo de forma análoga para o plano B, tem-se:

Plano B - Entrada de 600,00 + 5 de R \$126,00

Função que modela o plano B de financiamento,

$$
f(x)=4,024 x^{6}-5,024 x^{5}+1=0 .
$$

Na fase I fazendo $f(x)=0$, obteve-se,

$$
h(x)=4,024 x^{6} \text { e } k(x)=5,024 x^{5}-1
$$

Em seguida, esboçando os gráficos das funções $h(x)$ (cor, ciano) e $k(x)$ (cor, vermelha) (Gráfico 5), isolou-se o intervalo [1.02,1.2], definindo desta maneira a aproximação inicial da raiz sendo $x_{0}=1.2$. Posteriormente, aplica-se novamente a formulação matemática (31) estipulando uma precisão $\varepsilon=10^{-7}$, obtendo-se um valor para a raiz procurada $\xi_{B}=1.077062938412195$, que inserida em (16), obtém-se a taxa de juros $i_{B}=0,0770$ ou $i_{B}=7,70 \%$ ao mês (Gráfico 6). 
Gráfico 5 -Isolamento da raiz necessário para estimar a taxa de juros vinculada ao plano B de financiamento (computador)
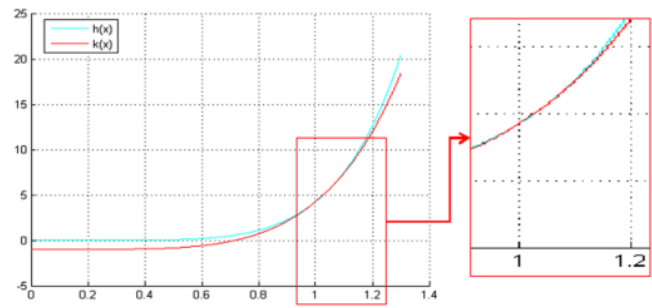

Fonte: Autoria própria
Gráfico 6 -localização da raiz vinculada a modelagem do plano B (computador)

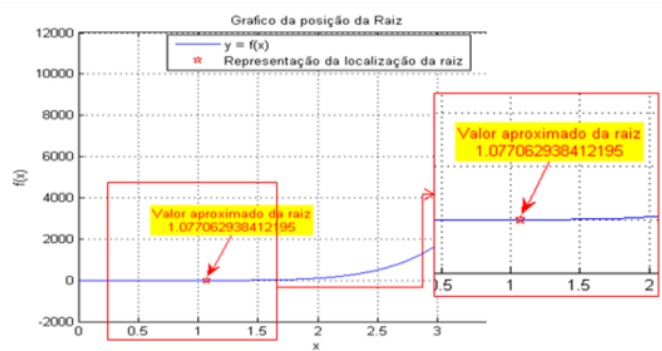

Fonte: Autoria própria

Utilizando de maneira similar a técnica para isolar um intervalo que contenha uma raiz, bem como a determinação da aproximação inicial para o método de NewtonRaphson ser inicializado para estimar a raiz procurada no caso do computador, nesta ordem localiza-se o intervalo [1.01,1.2] e $x_{0}=1.2$. Em seguida, utilizando (31) estimando a raiz $\xi_{A}=1.041090435578389$ (Gráfico 7 e Gráfico 8,respectivamente) para o micro-ondas. Portanto, inserindo o valor de $\xi_{A}$ em (16), obtém-se $i_{A}=4,10 \%$ ao mês.

Gráfico 7 - Isolamento da raiz necessário para estimar a taxa de juros vinculada ao plano A de financiamento (micro-ondas)
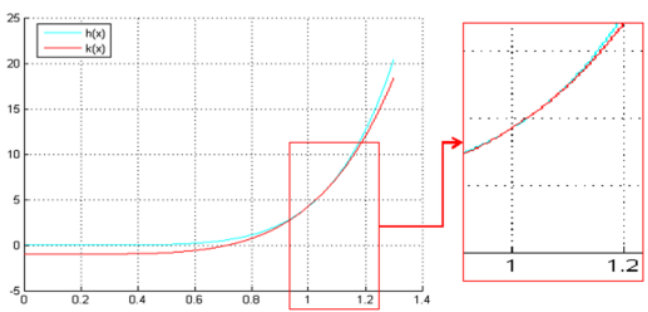

Fonte: Autoria própria
Gráfico 8-Localização da raiz vinculada a modelagem do plano A (micro-ondas)

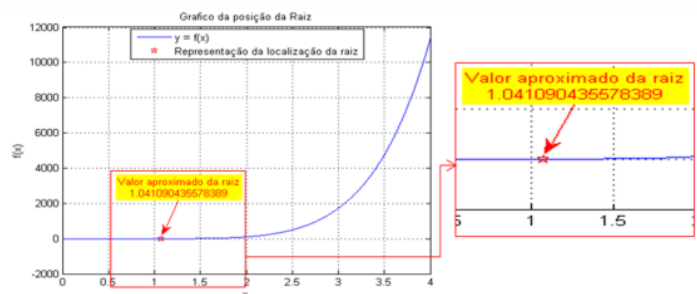

Fonte: Autoria própria

Plano B - Entrada de 300,00 + 4 de R $\$ 36,25$

Agora, utilizando o intervalo isolado [1.02,1.2] (Gráfico 9) e definindo a aproximaçãoinicial $\quad x_{0}=1.2$ para inicializar (31), calcula-se $\xi_{B}=1.164648489352564$ (Gráfico 10), que por conseguinte inserindo em (16), determina-se $i_{B}=16,46 \%$ ao mês. 
Gráfico 9 - Isolamento da raiz para estimar a taxa de juros ao plano $\mathrm{B}$ de financiamento (micro-ondas)
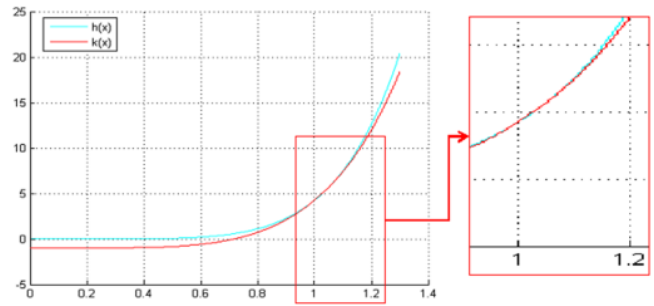

Fonte: Autoria própria
Gráfico 10 - Localização da raiz vinculada a modelagem do plano B (micro-ondas)
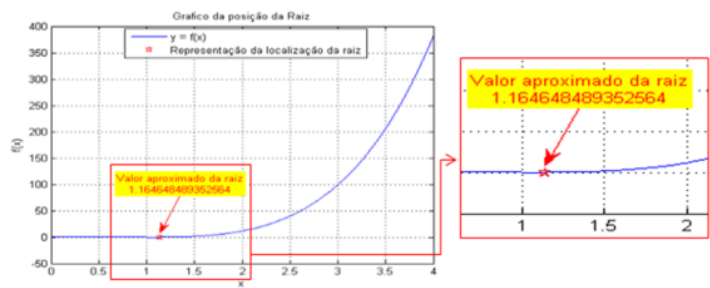

Fonte: Autoria própria

Por fim, utilizando os palano A comercializado para o eletroeletrônico televisão,

tém-se: o intervalo isolado [1.02,1.2] (Gráfico 11) com aproximação inicial $x_{0}=1,2$.

Assim, utilizando (31) determina-se $\xi_{A}=1.038648691598535$ (Gráfico 12) e substituindo

$\xi_{A}$ em (16), calcula-se $i_{A}=3,86 \%$ ao mês.

Gráfico 11- Isolamento da raiz para estimar a taxa de juros ao plano A de financiamento (televisão)

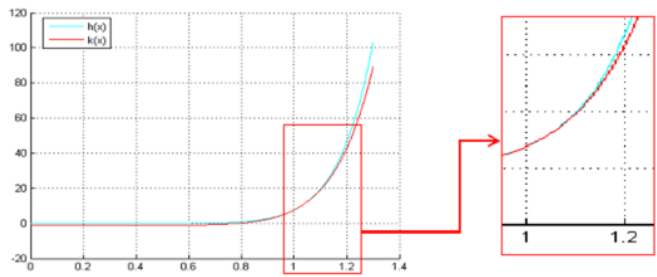

Fonte: Autoria própria
Gráfico 12 - Localização da raiz para a modelagem do plano A (televisão)

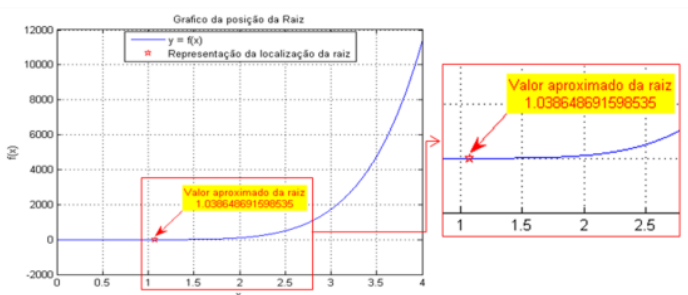

Fonte: Autoria própria

Em relação ao plano B (entrada de 500,00 + 9 de $\mathrm{R} \$ 64,55$ ), isolou-se o intervalo [1.02,1.2] (Gráfico 13), atrelado a um valor $x_{0}=1,2$ para novamente utilizar-se como inicializador da formulação (31), obtendo-se $\xi_{B}=1.039520987407710$ (Gráfico 14), tal valor foi inserido em (16) implicando em uma taxa de juros $i_{B}=3,95 \%$ ao mês.

Gráfico 13 - Isolamento da raiz para estimar a taxa de juros ao plano $\mathrm{B}$ de financiamento (televisão)

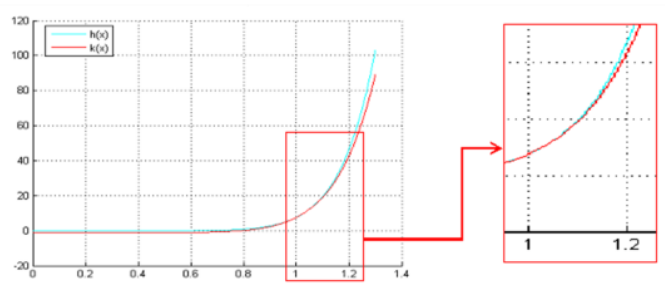

Fonte: Autoria própria
Gráfico 14 - Localização da raiz para a modelagem do plano B (televisão)

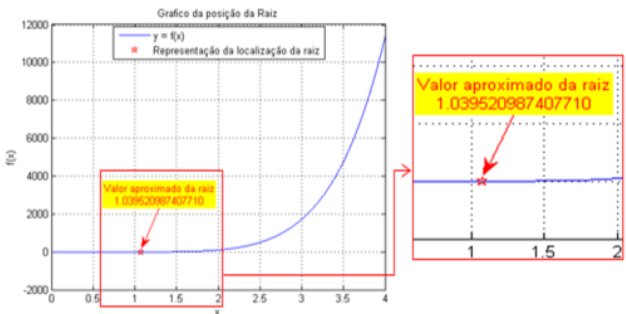

Fonte: Autoria própria 


\section{ANÁLISES DOS RESULTADOS DOS APARELHOS}

Para o computador, a priori, o plano B mostrou-se mais vantajoso que o plano A, pois o montante pago no plano B é de $\mathrm{R} \$ 1.230,00(600,00+5 \times 126,00=$ Montante $)$ sendo menor que o montante determinado pelo plano A, ou seja, $\mathrm{R} \$ 1.237,00(402,00+$ $5 \times 167,00=$ Montante). No entanto, este fato torna-se ilusório, pois os juros cobrados no plano B são de 7,70\% ao mês, enquanto que o estimado no plano A foi de $5,91 \%$ ao mês, constatando assim, que o plano A é mais vantajoso financeiramente para o consumidor. Em relação ao produto micro-ondas, verificou-se que tanto a análise através do montante, quanto a estratégia aqui apresentada informaram a mesma constatação, ou seja, o plano A indica a melhor escolha para o consumidor, visto que os juros vinculados ao plano B são de $16,46 \%$ ao mês, e ao plano A de 4,10\% ao mês.

Para o caso da televisão, se o consumidor utilizar somente o parâmetro montante para analisar os planos envolvidos, o mesmo efetivaria uma escolha errada, visto que o montante relacionado ao plano B $(\mathrm{R} \$ 1.080,95)$ é menor que o do plano A ( $\mathrm{R} \$ 1.098,94)$. Por outro lado, quando examina-se as taxas de juros obtidas pela estratégia da pesquisa a constatação inverte-se, ou seja, o plano A é mais vantajoso financeiramente para o consumidor.

\section{CONSIDERAÇÕES FINAIS E PERSPECTIVAS}

Neste trabalho, foi desenvolvido uma pesquisa de campo no centro comercial de Abaetetuba-PA, para coleta de preços necessarios para o desenvolvimento da problemática abordada. Sendo assim, foram obtidos planos de financiamentos nas lojas da referida cidade, os quais foram examinados por um modelo utilizado pelo PROCON, gerando uma equação que necessita de uma estratégia numérica para estimar uma raiz da mesma. Através dessa abordagem foi possível concluir qual plano é mais vantajoso financeiramente para o cliente, destacando que com apenas a informação do montante da dívida, o mesmo não garante uma escolha vantajosa para o consumidor, pois a informação determinante é a taxa de juros

Como perspectiva é indicado que haja um estudo mais aprofundado fazendo com que este trabalho se expanda em relação aos seus conceitos para situações em que o financiamento não fique somente restrito a prestações fixas. Fazendo isto, torna-se necessário a adaptação do modelo apresentado ou a apresentação um novo modelo matemático que 
seja capaz de representar este tipo de financiamento. Além disso, indica-se a implementação da estratégia abordada em uma interface que possa ser utilizada através de um aplicativo para celular tornando acessível ao público em geral.

\section{REFERENCIAS}

BARROSO, Leônidas Conceição et al. Interpolação. Cálculo numérico: (com aplicações). 2. ed. São Paulo: HARBRA, 1987.

CECCATO, Vanderlei; FRANCISCO, Reinaldo. Ensinando Matemática Aplicada ao Ensino Médio com o uso de Novas Metodologias. 2009. http://www.diadiaeducacao.pr.gov.br/portals/pde/arquivos/2462-8. Acesso em: $02 \mathrm{de}$ setembro de 2020.

FILHO, Benigno Barreto; SILVA, Cláudio Xavier da. Matemática: aula por aula. $1^{\text {a }}$ série. SP: FTD, 2003.

PUCCINI, Abelardo de Lima. Matemática Financeira: objetiva e aplicada com planilha eletrônica. RJ: LTC Editora, 1993.

STARK, Peter. Introdução aos métodos numéricos/Peter Stark; tradução de João Bosco Pitombeira de Carvalho. Rio de Janeiro: Interciência, 1979.

RUGGIERO, Márcia A. Gomes; LOPES, Vera Lúcia da Rocha. Interpolação. Cálculo numérico: aspectos teóricos e computacionais. 2. ed. São Paulo: Person Makron Books, 1996.

\section{Recebido em: 10/10/2021}

Aprovado em: 10/11/2021 